\title{
Light-in-flight digital holography display
}

\author{
H. Rabal, J. Pomarico, and R. Arizaga
}

We present a digital speckle-pattern interferometric setup that can be operated at TV frame rates ( $30 \mathrm{~ms}$ ) to display the locus of the points at which the optical-path difference between the reference and object beams is within the coherence length. Experimental results are shown.

Key words: Light in flight, digital speckle-pattern interferometry.

In 1978 Abramson $^{1}$ demonstrated a method called light-in-flight holography to display the locus of the zero optical-path difference (OPD) between the object and the reference beams of a holographic setup. $\mathrm{He}$ used the short coherence length of an argon-ion laser without an intracavity étalon. A picosecond laser was also used for this purpose. ${ }^{2}$ A similar method had been proposed by Boden et al. ${ }^{3}$ and Denisyuk et $a l .4$

On the other hand, digital speckle-pattern interferometry (DSPI) has developed as a powerful tool to perform several operations usually associated with holographic interferometry in the image plane; thus this method is also called digital holography. Deformation measurements and generation of contours of three-dimensional objects are some examples., 5 A review of some of these applications can be found in Ref. 7, but a profusion of recent developments exists in the literature.

In its basic form the image of an object with a superposed coherent background is registered by a TV camera and then digitized and stored in a frame memory. Subsequent images are subtracted from the initial image, and the square or the modulus of the difference is shown on a monitor. A phase change between the reference and the object waves is introduced between the first and the final observed states. It can be produced, for example, by a deformation of the object or by a change in the illumination conditions. If this phase difference on the image is slowly varying on areas comprising several pixels,

The authors are with the Centro de Investigaciones Opticas, Casilla de Correo 124, La Plata, 1990, Argentina.

Received 12 March 1993; revised manuscript received 19 November 1993.

0003-6935/94/204358-03\$06.00/0.

(1) 1994 Optical Society of America. then the same speckle pattern is present in both images in regions in which the phase difference is $2 \pi$ or congruent values. In these regions, subtraction produces a dark area in the result. Other phase delays modify the resulting speckle pattern, and the subtraction gives a nonzero response. Fringes are thus obtained in the subtracted image showing the locus of constant phase difference. High resolution inherent to analog holography is not required here. Only the speckle grains must be barely resolved by the imaging system. Three-dimensional image formation is sacrificed, but operation at TV frame rates without chemical developing is obtained as a trade.

We describe now how a DSPI experiment can be modified to obtain a light-in-flight display. Figure 1 shows the experimental setup. A cw argon-ion-laserpumped Rhodamine 110 dye laser is employed $(\bar{\lambda}=$ $550 \mathrm{~nm}$ ). A piezoelectrically driven mirror (PEM) is used to introduce a $\pi$-phase shift in the reference beam between the two exposures. Lens $L$ conjugates the focal point of the beam expander, $\mathrm{E}$, in the front focal plane of the imaging system of the CCD camera, thus producing a plane wave on the detector array. The image registered by the detector is the superposition of a speckle pattern and a uniform background produced by the reference beam.

For all image places in which the OPD is greater than the coherence length of the light the phase shift is of no consequence. At these points the superposition is incoherent; therefore a phase shift in the reference beam does not change the measured intensity, and subtraction produces a dark output at these points.

In regions in which the superposition is coherent, interference fringes are produced. These fringes are substituted by the complementary ones when the phase shift is introduced and the observed speckle pattern changes. Though the actual fringes are not resolved by the TV camera, subtraction in these 


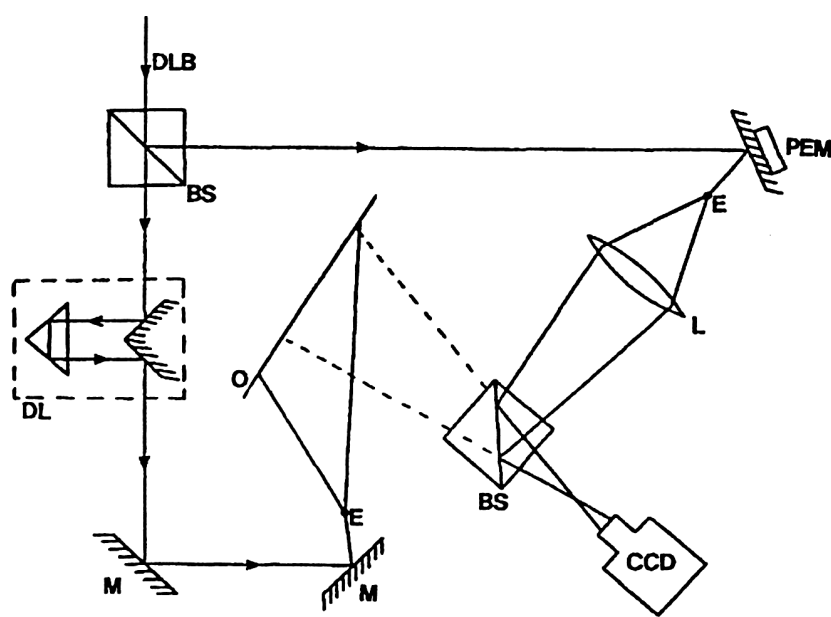

Fig. 1. Experimental setup: DLB, dye laser beam; BS's, beam splitters; M's, mirrors; E's, beam expanders; $O$, plane object; $L$, lens; PEM, piezoelectrically driven mirror; DL, delay line (optional); CCD, TV camera connected to a frame grabber a and computer.

regions produces a nondark result depicting the locus at which the OPD is within the coherence length.

A delay line (DL) in one of the beams can be used to change the place at which the object wave front differs in optical path with the reference wave front by less than the coherence length, thus producing a different contour. It can be seen that a sharp transition from coherent to incoherent in the light source as a function of OPD is desirable for a well-defined intersection of the wave front with the object.

Usually a spherical reference beam emerging from the center of the diaphragm is used to illuminate the whole image in DSPI systems. This configuration also leads to a light-in-flight display. Nevertheless, using a plane wave a as reference beam is more appropriate because then all of the path difference in the interference pattern can be attributed to the object beam. Only then can the display show the propagation of the object wave accurately.

The $f$-number of the camera lens was set at a relatively high value $(f=11)$ so that speckle grains could be resolved by the CCD array. This geometry introduces a certain amount of vignetting, but with a long enough focal length (in our case, $135 \mathrm{~mm}$ ) in the imaging lens, the best part of the CCD detector area (roughly $1 \mathrm{~cm} \times 1 \mathrm{~cm}$ ) can be illuminated.

As the experiment is performed in the image plane, it is not necessary to use an object-to-reference-beam intensity ratio greater than 1 . This ratio was used in our experiment.

The geometrical aspects of this experiment can be easily understood in terms of the holodiagram. ${ }^{8}$ The two foci of the ellipsoids are in this case the focal point of $E$, the origin of the beam illuminating the object, and the center of the image-forming lens (Fig. 1).

Figure 2 shows the experimental result obtained for the intersection of a spherical wave front with a plane object. The holodiagram constants for this

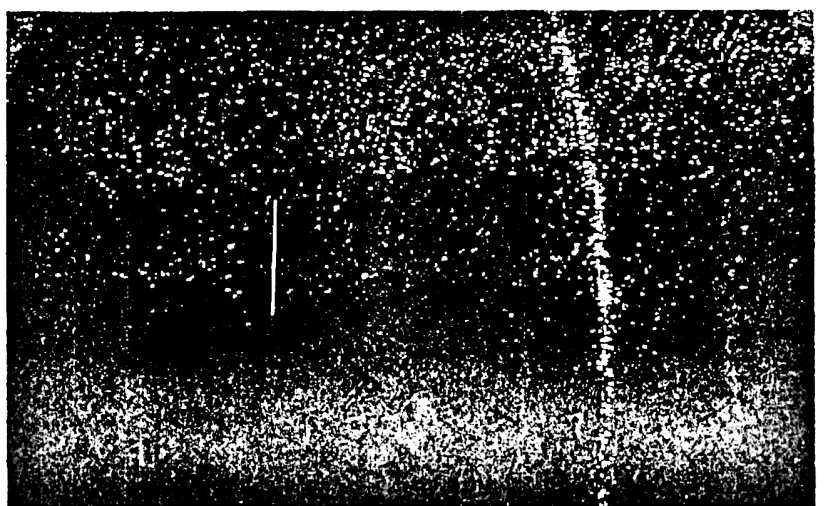

Fig. 2. Intersection of a spherical wave front with a plane-tilted object.

experiment are $k \cong 1.15\left(\alpha \cong 30^{\circ}\right)$ and $k^{*}$ nominally infinite $(\beta \equiv 0)^{2=8} ; 2 \alpha$ is the angle between illumination point $E$ and the observation point defined by the center of the lens as seen from a generic object point, and $2 \beta$ is the angle between the object beam and the reference beam at the $\mathrm{CCD}$ array plane.

A small parallel glass plate was then introduced in the central region of the object, and the wave front showed its presence by a delay corresponding to $\sim 4$ ps. The results are shown for two different positions of the delay line in Fig. 3.

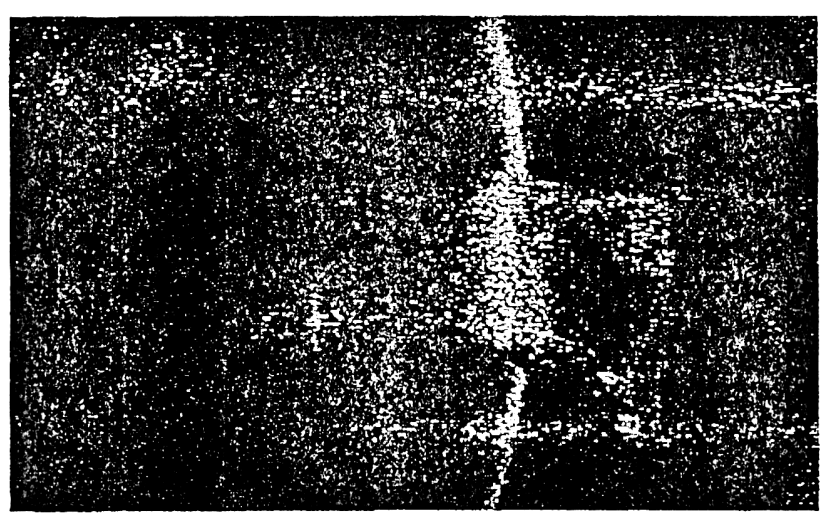

(a)

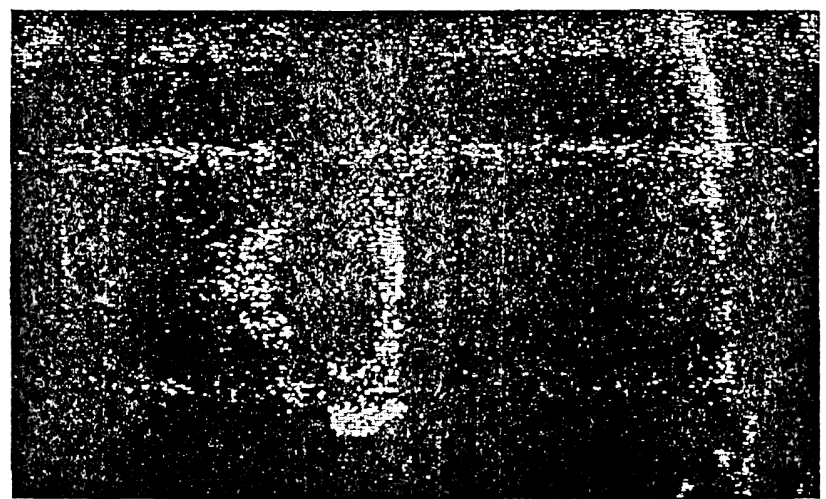

(b)

Fig. 3. Intersection of a spherical wave front with the same object after passing through a small parallel glass plate for two different positions of the delay line. 
An adequately designed Michelson interferometer acting simultaneously as a beam splitter and as a delay line could be used to obtain two bright lines. A Fabry-Perot interferometer could also be used to increase the number of lines.

We have proposed and demonstrated a DSPI experiment to depict the locus of the intersection of an object with an evolving wave front. Although it is intrinsically a sequential method, it can be implemented at a TV frame rate without chemical processing of a holographic plate. The position of the wave front can be changed by the adjustment of a delay line.

This work was supported by Consejo Nacional de Investigaciones Científicas y Técnicas grant PID 3-071700/88 and a grant from Fundación Antorchas. We thank the Laser Group of the Centro de Investigaciones Opticas for providing the dye laser. J. Pomarico is a Fellow of the Comisión de Investigaciones Científicas de la Provincia de Buenos Aires.

\section{References and Notes}

1. N. Abramson, "Light-in-flight recording by holography," Opt. Lett. 3, 121-123 (1978).

2. N. Abramson, "Light-in-flight recording: high-speed holographic motion pictures of ultrafast phenomena," Appl. Opt. 22, 215-232 (1983).

3. F. Boden, H.-D. Reidenbach, and G. Köppen-Borgstedt, "Gated viewing by holographic range gating," Optik 51, 199-212 (1978).

4. Yu. N Denisyuk and D. I. Staselko [Appl. Opt. 31, 1682-1684 (1992)] claimed that the method was previously developed by them in 1969.

5. J. Butters, R. Jones, and C. Wykes, "Electronic speckle pattern interferometry," in Speckle Metrology, R. Erf, ed. (Academic, New York, 1978), pp. 111-158.

6. N. Bolognini, H. Rabal, and R. Torroba, "Single-beam digital holographic contouring," Appl. Opt. 31, 1009-1011 (1992).

7. J. R. Tyrer, "A critical review of recent developments in electronic speckle pattern interferometry," in Holographic Nondestructive Testing, C. M. Vest, ed., Proc. Soc. Photo-Opt. Instrum. Eng. 604, 95-99 (1986).

8. N. Abramson, "The holo-diagram. VI. Practical device in coherent optics," Appl. Opt. 11, 2562-2571 (1972). 